\title{
Observations on the Differentiation of Plasmodia into Fruiting Bodies by the True Slime Mould, Didymium nigripes
}

\author{
By S. LUCAS, M. RAZIN AND N. KERR \\ Department of Zoology, University of Minnesota, \\ Minneapolis, Minnesota 55455, U.S.A. \\ (Accepted for publication 4 March 1968)
}

\begin{abstract}
SUMMARY
Under defined experimental conditions plasmodia of Didymium nigripes began to differentiate into fruiting bodies $\mathrm{I} 6-\mathrm{I} 8 \mathrm{hr}$ after transfer to bacteriafree agar. The process has been divided into a number of easily recognizable stages and the duration of each stage determined. Plasmodia lost ability to continue vegetative growth at a time before the actual fruiting bodies began to form.
\end{abstract}

\section{INTRODUCTION}

The mature fruiting body of the true slime mould Didymium nigripes consists of a rounded black spore-mass supported above the substrate on the apex of an acellular stalk. Under defined conditions (Kerr \& Sussman, 1958) the spores germinate and give rise to uninucleate myxamoebae which, when plated with a bacterial food source, feed and multiply by binary fission. After a period of exponential growth, the myxamoebae differentiate into plasmodia which grow to form macroscopic multinucleate networks of rhythmically streaming protoplasm. Plasmodia can be maintained as such by subculturing them every second day to agar plates which have been spread with bacteria. They move across the agar as they grow, engulfing the bacteria. If they are not subcultured before they reach the far side of the plate, they usually sweep back and forth across the plate about one and one-half times before starving sufficiently to differentiate into fruiting bodies. When a plasmodium is transferred to bacteria-free agar so that the loss of its bacterial food supply is more abrupt, the differentiation into fruiting bodies is more uniformly timed. The purpose of the present study was to divide the morphogenesis of plasmodia to fruiting bodies into stages and to determine the timing of each stage. The point at which plasmodia were no longer able to resume vegetative growth even in the presence of a bacterial food source was also determined. Such information is a necessary prerequisite to the study of the differentiation at a molecular level.

\section{METHODS}

Didymium nigripes was grown in monoxenic culture with Aerobacter aerogenes on dilute glucose peptone yeast-extract (GPY/5) agar as previously described (Kerr \& Sussman, 1958). Plasmodia were maintained as such by subculturing a $1 \mathrm{~cm} .{ }^{2}$ piece every 2 days to GPY $/ 5$ agar which had been spread with $A$. aerogenes 2 days earlier.

To induce fruiting, plasmodia which had advanced one-half to three-quarters of the way across a GPY $/ 5$ agar plate were transferred to bacteria-free $1 \cdot 5 \%$ Ionagar no. 2 buffered with 0.01 M-phosphate ( $\mathrm{pH} \mathrm{6.6)}$. Cylinders $(3.5 \mathrm{~mm}$. diam.) which had been 
cut from the solid protoplasmic region of the advancing plasmodial fronts with a sterile brass cork-borer were transferred by using a hoe-like nichrome wire transfer tool, which enabled restoration of the original size and orientation of the plasmodial discs on the agar.

At $30 \mathrm{~min}$. intervals the morphology of each transfer was noted. After a period of time each starving plasmodium was tested to see whether, at that time, it was committed to fruiting, by selecting its morphologically most advanced part and transferring it back to GPY/5 agar spread with Aerobacter aerogenes. The desired part of the plasmodium, along with the underlying agar, was cut out with a $\mathrm{I} \mathrm{cm} .^{2}$ nichrome cutter and inverted on the bacterial agar, so that the plasmodium was in contact with the lawn of bacteria; $24 \mathrm{hr}$ later each transfer had fruited or grown as a plasmodium. All material was incubated at $23^{\circ}$.

Time-lapse cine films were made, in black and white and in colour with high-speed Ektachrome type B, using a Bolex H I6 Reflex camera, of both the top and side views of fruiting plasmodia (Kerr, I965). Sequences were taken with the use of extension tubes between the camera and lens, and through a Zeiss microscope using a $\times 2.5$ objective and an $\times 8$ ocular.

\section{RESULTS AND DISCUSSION}

During the morphogenesis of plasmodia into fruiting bodies on bacteria-free agar, the following stages were distinguished.

Advancing front stage. During the first $\mathrm{I} 6-\mathrm{I} 8 \mathrm{hr}$ after it had been transferred to nonnutrient agar, the starving plasmodia continued to migrate across the agar ( $\mathrm{Pl}$. I, fig. I). The duration of this stage was the most variable part of fruiting, being dependent on the time since subculture of the parental plasmodia. The use of plasmodia which had migrated three-quarters of the way across the GPY/5 agar plate instead of one-quarter of the way shortened this stage by several hours.

Accumulation stage. The plasmodial fronts stopped their advance and increased in area to form broad accumulations of protoplasm which were as thick as a large plasmodial vein. The veins flowing into such accumulations gradually lost all of their protoplasm to them as they dried and became the hypothallus (Pl. I, fig. 2).

Mounding stage. Ridges of protoplasm pushed up in the accumulations. Each ridge gradually subdivided into mounds of unequal size which were usually connected to one another by single veins of streaming protoplasm (Pl. I, fig. 3). The streaming between the mounds gradually slowed and then stopped as all of the protoplasm entered the mounds. The last amounts of protoplasm to enter the mounds became lumpy and flowed in discrete pieces.

When mounds were manipulated with a microneedle, their structural strength was greater than that of a plasmodial vein. This might have been due either to the mounds becoming surrounded by a stronger membrane than that around the plasmodial veins, or to a stiffening of the protoplasm in the mounds.

Rising stage. The protoplasm in the mounds rose above the substrate to form pulsating peg-like structures (Pl. I, fig. 4). After each forming fruit had reached about two-thirds of its final height, the acellular stalk began to become visible. The protoplasm which was to form the stalk constricted around the more internal protoplasm in a progressive fashion, beginning first at the bottom, so that eventually all of the more internal protoplasm was squeezed out at the top of the forming stalk. In the 
time-lapse films the protoplasm, presumably as a continuation of the rhythmic streaming characteristic of plasmodia, continued to pulsate while it rose off the substrate.

White fruit stage. The protoplasm in the head of the fruit continued to pulsate for about an hour after the completion of the stalk. The fruit remained colourless, like the plasmodium, for several hours.

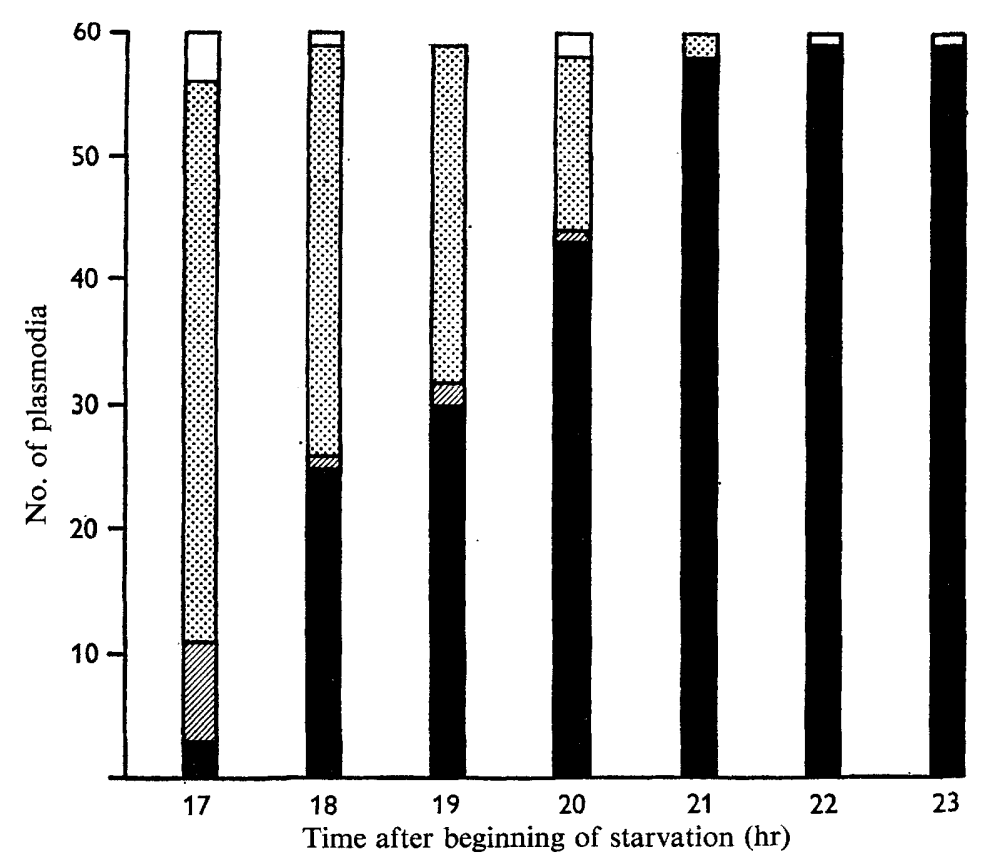

Fig. I. Didymium nigripes: the commitment of plasmodia starved for varying periods of time to complete the fruiting process. $\square$, Plasmodia neither grew vegetatively nor fruited; $\square$, the entire plasmodium resumed vegetative growth; 图, a portion of the plasmodium fruited, the remainder resuming vegetative growth; $\boldsymbol{m}$, the entire plasmodium differentiated into fruiting bodies.

Table I. Didymium nigripes: timing of the fruiting sequence

\begin{tabular}{lc}
\multicolumn{1}{c}{ Stage } & Duration (hr) \\
\pm I S.E.
\end{tabular}

Pigment synthesis stage. The pale-white fruiting body slowly turned pink. The colour gradually deepened in intensity to a brown, and finally to black, the colour of the mature fruiting body. This change in colour is due to the synthesis of a melaninlike material which is a structural element of the spore walls (personal communication from E. Haskins).

Through the use of the standardized transfer technique, all transfers from a single 
plasmodium went through the above stages in relative synchrony. The average time spent in each stage by 60 transfers from at least 12 plasmodia, transferred on three separate days, is given in Table $\mathrm{I}$. To determine the time after which starving plasmodia could no longer revert to the vegetative state when placed in contact with a bacterial food supply, at timed intervals plasmodia were returned to GPY/5 agar spread with bacteria. The results are shown in Fig. I. Three groups of 20 plasmodia were tested after each hour of starvation. Between 17 and $2 \mathrm{I} \mathrm{hr}$ of starvation the percentage of plasmodia which were committed to form fruiting bodies increased steadily.

From the data given in Table $I$ it can be seen that almost all of the variability in the time of fruiting occurred in the advancing front stage, which lasted I6-I $8 \mathrm{hr}$ after the transfers to non-nutrient agar. Since the total time from the beginning of starvation to the completion of pigment synthesis was from 32 to $34 \mathrm{hr}$, this represents a variation of only $6 \%$ in the time of fruiting. This variation almost certainly was due to minor differences in the distance the parental plasmodium had grown across the GPY/5 agar plate before portions of plasmodium were transferred to starvation conditions. Pieces of plasmodium ( $\left(\mathrm{cm} .^{2}\right.$ ) were subcultured to one side of GPY $/ 5$ agar plates which had been spread with bacteria 2 days earlier. The plasmodia spent about 2 days moving across such a plate. Since plasmodia can ingest bacteria only at their leading edge, it is obvious that a recently subcultured plasmodium has substantially greater feeding interface per unit of protoplasmic mass than does a plasmodium which is more than half way across a growth plate. The number of undigested bacteria in food vacuoles must also be greater per unit of protoplasm in a recently subcultured plasmodium. If equal amounts of recently subcultured plasmodium or advanced plasmodia are transferred to plain agar, one would expect the advanced plasmodium to starve more quickly than the recently subcultured plasmodium. Hence the $2 \mathrm{hr}$ variation in the length of the advancing front stage almost certainly represented minor differences in the nutritional state of the parental plasmodia.

A comparison of the data given in Table I with Fig. I indicates that advancing front stages were not yet irreversibly committed to form fruiting bodies. The mounding stage did not begin until 19.4 to $21.4 \mathrm{hr}$ after the beginning of starvation, by which time over $60 \%$ of the plasmodia were already irreversibly committed to differentiate into fruiting bodies. The irreversible commitment to form fruiting bodies must, therefore, have taken place during the accumulation stage, at a time well before the actual site of fruiting body formation had been determined. This represents an example of a case in which differentiation precedes morphogenesis in a clear-cut manner. Although the data presented here were obtained from small, carefully chosen pieces of plasmodium, the various stages can easily be recognized under conditions where larger amounts of protoplasm, sufficient for analysis by techniques such as acrylamide gel electrophoresis, can be obtained.

This work was supported by grant no. AI-0552 I from the National Institutes of Health, USPHS.

\section{REFERENCES}

Kerr, N. S. (1965). Didimium nigripes, a true slime mould (I6 mm. film). Progress in Protozool. Int. Congr. Series no. 91, 276. Amsterdam: Excerpta Medica Foundation.

Kerr, N. S. \& Sussman, M. (1958). Clonal development in the true slime mould, Didymium nigripes. J. gen. Microbiol. 19, 173 . 


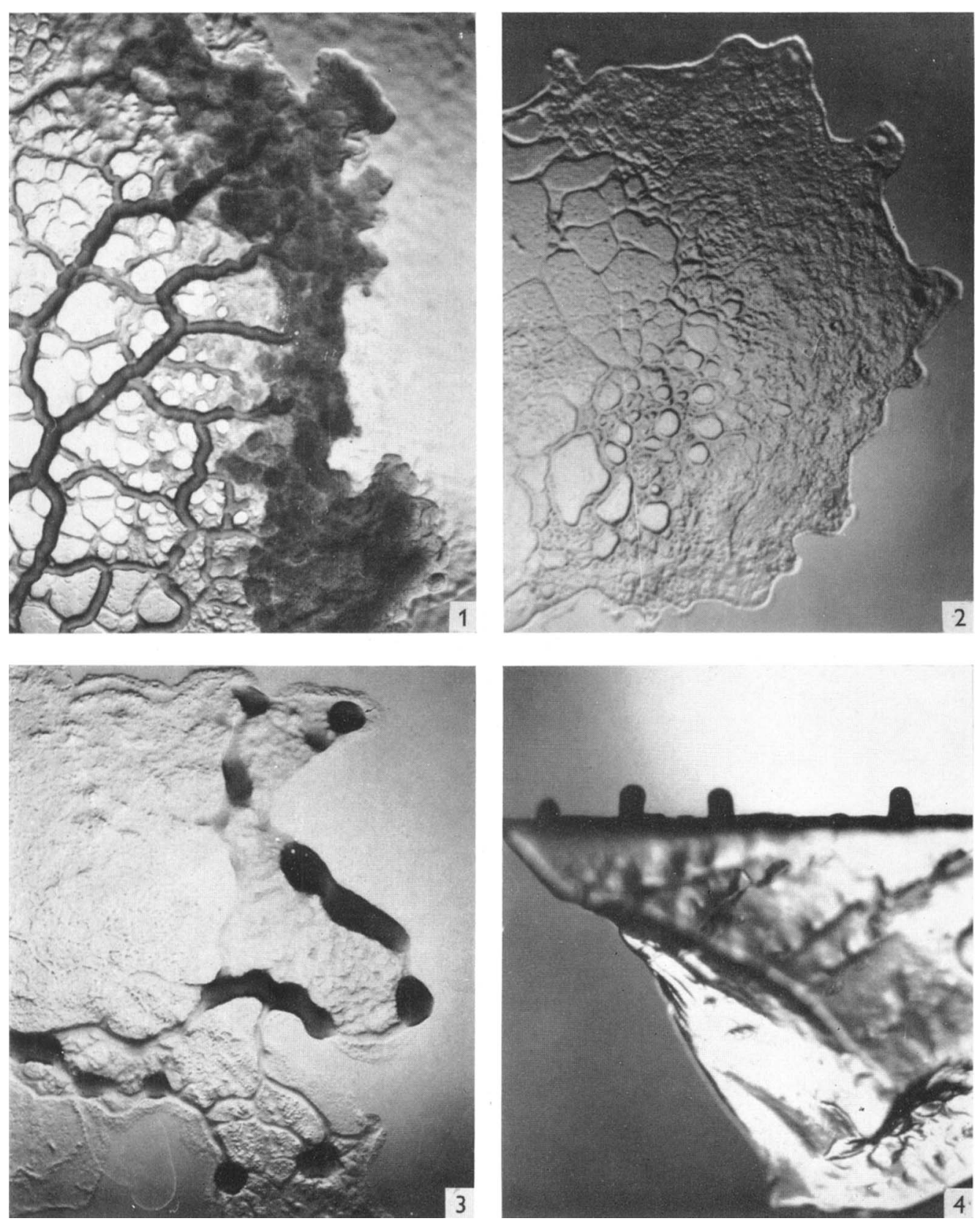
EXPLANATION OF PLATE

Didymium nigripes: stages in the differentiation of plasmodia into fruiting bodies $\times 10$.

Fig. I. The advancing front stage. The plasmodium is still advancing across the agar.

Fig. 2. The accumulation stage. The plasmodium has ceased to advance across the agar and an accumulation of protoplasm has formed.

Fig. 3. The mounding stage. Mounds, still connected to one another by veins of streaming protoplasm, appear within the accumulation.

Fig. 4. The rising stage, side view. The presumptive fruiting bodies are shaped as the mounds rise above the agar surface. 